TRANSACTIONS OF THE

AMERICAN MATHEMATICAL SOCIETY

Volume 361, Number 10, October 2009, Pages 5089-5109

S 0002-9947(09)04540-1

Article electronically published on May 6, 2009

\title{
FROM FAST TO VERY FAST DIFFUSION IN THE NONLINEAR HEAT EQUATION
}

\author{
NOUREDDINE IGBIDA
}

\begin{abstract}
We study the asymptotic behavior of the sign-changing solution of the equation $u_{t}=\nabla \cdot\left(|u|^{-\alpha} \nabla u\right)+f$, when the diffusion becomes very fast, i.e. as $\alpha \uparrow 1$. We prove that a solution $u_{\alpha}(t)$ converges in $L^{1}(\Omega)$, uniformly for $t$ in subsets with compact support in $(0, T)$, to a solution of $u_{t}=\nabla \cdot\left(|u|^{-1} \nabla u\right)+f$. In contrast with the case of $\alpha<1$, we prove that the singularity 0 created in the limiting problem, i.e. $\alpha=1$, is an obstruction to the existence of signchanging solutions. More precisely, we prove that, for each $t \geq 0$, the limiting solutions are either positive or negative or identically equal to 0 in all $\Omega$. This causes the limit to be singular, in the sense that a boundary layer appears at $t=0$, when one lets $\alpha \uparrow 1$.
\end{abstract}

\section{1.. INTRODUCTION AND MAIN RESULTS}

In this paper we study the asymptotic behavior of the sign-changing solutions of the equation

$$
u_{t}=\nabla \cdot\left(|u|^{-\alpha} \nabla u\right)+f
$$

as $\alpha \uparrow 1$.

Equation (1.1) with $\alpha<0$ is the so-called porous medium equation. Existence, uniqueness, asymptotic behavior as the diffusion is very slow, i.e. $\alpha \downarrow-\infty$, and many other properties have been extensively studied for this equation. If $0 \leq \alpha<1$, (1.1) is the nonlinear fast diffusion equation, since it is a particular case of the general formulation $u_{t}=\nabla \cdot(D(u) \nabla u)+f$ with the diffusivity $D(u)$ satisfying $D(u) \rightarrow+\infty$ as $u \rightarrow 0$, though it must be borne in mind that $D(u) \rightarrow 0$, as $u \rightarrow \infty$. The study of the peculiar case $0<\alpha \leq 1$ is also motivated by some physical applications. For instance, equation (1.1) arises in plasma physics, the kinetic theory of gases and solid state (cf. [8, 24] and 21]). It also appears in Riemannian geometry to describe the evolution of a conformal metric in the plane under Ricci flow (cf. [15], 30, 29]). The reader can also see papers [2], 26] and [27] for extensive references on equation (1.1).

The study of the limit as $\alpha \uparrow 1$ is a part of a wide program for understanding the singular limit phenomena for evolution problems. Indeed, for $\alpha$ very close to 1 , a self-organizer process appears in the equation and makes the limiting problem

Received by the editors February 4, 2005 and, in revised form, September 19, 2006, February 9, 2007 and March 12, 2007.

2000 Mathematics Subject Classification. Primary 35K60, 35K65, 35B40.

Key words and phrases. Singular limit, fast diffusion, logarithmic diffusion equation, degenerate parabolic equation, nonhomogeneous Neumann boundary condition, porous medium equation, sign-changing solution, boundary layer, semigroup of contraction.

(C)2009 American Mathematical Society 
strikingly different. For other singular limits for (1.1), one can also see papers 11$]$ and [3] for $\alpha \downarrow-\infty$ and paper [25] for $\alpha \rightarrow 0$. In [27, the author discusses the fast and superfast cases of (1.1) which correspond to $0<\alpha<2$.

In this paper, we focus our attention on the case $\alpha=1$, since even if this case is a borderline of the case $\alpha<1$, the properties of the solutions in both cases are completely different. For instance, as we show, in contrast with $\alpha<1$, for which the existence of solutions holds for every $L^{1}$ initial data, the existence of a solution when $\alpha=1$ holds only with an additional sign condition on the initial data. Moreover, in contrast with the case $\alpha<1$, in which for any $t>0$, a solution $u(t)$ may vanish in a subregion of $\Omega$ and changes sign, in case $\alpha=1$, for any $t>0$, either $u(t)>0$ in $\Omega$ or $u(t)<0$ in $\Omega$ or $u(t) \equiv 0$ in $\Omega$. This is strongly connected with the fact that equation (1.1) may be written in the standard form

$$
u_{t}=\Delta \varphi_{\alpha}(u)+f,
$$

where $\varphi_{\alpha}$ is given by

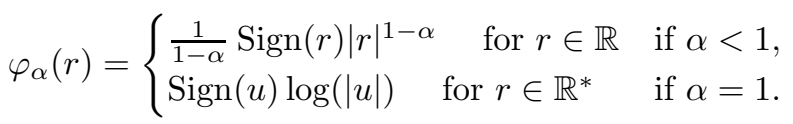

Note that if $\alpha<1$, then $\varphi_{\alpha}$ is a continuous increasing function in $\mathbb{R}$. On the other hand, when $\alpha=1$, the graph of $\varphi_{1}$ is a union of two graphs of continuous increasing functions in $\mathbb{R}$ and singular at 0 . The singularity of $\varphi_{1}$ at 0 seems to create an obstruction to the existence of sign-changing solutions. For the asymptotic behavior of a solution of (1.1), as $\alpha \uparrow 1$, one expects that a solution $u_{\alpha}$ of (1.1), for $\alpha<1$, converges to a solution corresponding to $\alpha=1$. However, due to the obstruction phenomena at 0 , the limit is singular in the case where $u_{0}$ is a sign-changing function and a boundary layer appears when one reaches the limit. In the case of $N=1$, this problem was studied in [28] in connection with the $(1-\alpha)$-Laplacian operator in $\mathbb{R}$. In that paper, the authors pointed out the obstruction phenomena at 0 for the limiting problem. Papers [13] and [17] treat the cases $N=1,2$ when the initial data is nonnegative. In this paper, we will prove convergence results of solutions $u_{\alpha}$ of (1.1), as $\alpha \uparrow 1$, and show how the boundary layer appears with respect to the average of the initial data and $f$, and also with respect to the average of the flux of the solution on the boundary.

Remember that, in $\mathbb{R}^{N}$, and in contrast with the case $\alpha<1$, 1.1) can be ill posed if $\alpha=1$, because of the nonuniqueness of the solution (cf. 12]). However, if $N=1,2$, Esteban, Rodriguez and Vazquez show in a series of papers (see the last paper [13] and the references therein) that the problem is well posed under additional conditions on the flux at infinity. For instance, a unique nonnegative radial symmetric solution exists in case $N=2$ with nonnegative radial symmetric initial data $u_{0} \in L^{1}\left(\mathbb{R}^{2}\right)$ and Neumann boundary condition at infinity described by

$$
\lim _{r \rightarrow \infty} \frac{r u_{r}(x, t)}{u(x, t)}=-g(t),
$$

where $g \geq 2, r=|x|$ and $u_{r}$ is the derivative of $u$ along the radial direction. In other words, if $\alpha=1$, one must consider (1.1) with some nonstandard boundary conditions in $\mathbb{R}^{N}$ of Neumann type. This approach was used in [16] to construct again nonnegative solutions of (1.1) for $\alpha=1$, as limits of solutions in a ball $B(R)$, of radius $R>0$, with nonhomogeneous Neumann boundary condition on $\partial B(R)$, by letting $R \rightarrow \infty$. 
In this paper, we consider equation (1.1), in a smooth bounded domain $\Omega$, with nonhomogeneous time dependent boundary condition on $\Gamma$, the boundary of $\Omega$,

$$
u^{-\alpha} \nabla u \cdot \vec{n}=g,
$$

where $\vec{n}$ denotes the outward normal of $\Gamma, g \in L^{2}(\Sigma)$ and $\Sigma=(0, T) \times \Gamma$. So, throughout the paper we denote by $E_{\alpha}\left(u_{0}, f, g\right)$ the following evolution problem:

$$
E_{\alpha}\left(u_{0}, f, g\right)= \begin{cases}u_{t}=\nabla \cdot\left(u^{-\alpha} \nabla u\right)+f & \text { in } Q=(0, T) \times \Omega, \\ u^{-\alpha} \nabla u \cdot \vec{n}=g & \text { on } \Sigma=(0, T) \times \Gamma, \\ u(0, .)=u_{0}(.) & \text { in } \Omega .\end{cases}
$$

We begin by a more or less known result concerning the existence and uniqueness of a solution of $E_{\alpha}\left(u_{0}, f, g\right)$, for $\alpha<1$.

Proposition 1. For any $\alpha<1$, if $u_{0} \in L^{2-\alpha}(\Omega), f \in L^{2}(Q)$ and $g \in L^{2}(\Sigma)$, there exists a unique solution $u$ of $E_{\alpha}\left(u_{0}, f, g\right)$ in the sense of

$$
\left\{\begin{array}{l}
u \in \mathcal{C}\left([0, T) ; L^{1}(\Omega)\right), \varphi_{\alpha}(u) \in L^{2}\left(0, T ; H^{1}(\Omega)\right), u(0)=u_{0} \text { a.e. } \Omega, \\
\frac{d}{d t} \int_{\Omega} \xi u+\int_{\Omega} \nabla \varphi_{\alpha}(u) \cdot \nabla \xi=\int_{\Gamma} \xi g+\int_{\Omega} \xi f \quad \text { in } \mathcal{D}^{\prime}(0, T), \forall \xi \in \mathcal{C}^{1}(\bar{\Omega}) .
\end{array}\right.
$$

For $\alpha=1$ and due to the nature of the graph of $\varphi_{1}$, we introduce the following subset of $L^{p}(\Omega)$ for $1 \leq p \leq \infty$ :

$$
\begin{aligned}
& L^{p}(\Omega)^{+}=\left\{u \in L^{p}(\Omega) ; u \geq 0 \text { a.e. } \Omega\right\}, \\
& L^{p}(\Omega)^{-}=\left\{u \in L^{p}(\Omega) ; u \leq 0 \text { a.e. } \Omega\right\}
\end{aligned}
$$

and

$$
L^{p}(\Omega)^{ \pm}=L^{p}(\Omega)^{-} \cup L^{p}(\Omega)^{+} .
$$

As common when dealing with purely Neumann boundary conditions, the quantity

$$
\mu(t)=\int_{\Omega} u_{0}+\int_{0}^{t}\left(\int_{\Gamma} g+\int_{\Omega} f\right) \quad \text { for any } t \in[0, T)
$$

plays a crucial role in the study of $E_{1}\left(u_{0}, f, g\right)$. We are going to use it extensively throughout the following subintervals (possibly empty) of $[0, T)$ :

$$
\begin{aligned}
I & =\{t \in[0, T) ; \mu(t)=0\}, \\
I^{+} & =\{t \in[0, T) ; \mu(t)>0\}, \\
I^{-} & =\{t \in[0, T) ; \mu(t)<0\}
\end{aligned}
$$

and

$$
I^{ \pm}=I^{+} \cup I^{-}
$$


Theorem 1. For any $u_{0} \in L^{p}(\Omega)^{ \pm}$, with $p>1, f \in L^{2}(Q)$ and $g \in L^{2}(\Sigma)$, there exists a unique solution $u$ of $E_{1}\left(u_{0}, f, g\right)$ in the following sense:

$$
\left\{\begin{array}{l}
u \in \mathcal{C}\left([0, T) ; L^{1}(\Omega)^{ \pm}\right), u(0)=u_{0} \text { a.e. } \Omega, u(t) \equiv 0 \text { in } \Omega, \text { for any } t \in I, \\
u(t) \mu(t)>0 \text { a.e. in } \Omega, \text { for any } t \in I^{ \pm}, \log (|u|) \in L^{2}\left(I^{ \pm} ; H^{1}(\Omega)\right), \\
\frac{d}{d t} \int_{\Omega} \xi u+\int_{\Omega} \nabla \varphi_{1}(u) \cdot \nabla \xi=\int_{\Gamma} g \xi+\int_{\Omega} f \xi \quad \text { in } \mathcal{D}^{\prime}\left(t_{1}, t_{2}\right), \\
\forall \xi \in \mathcal{C}^{1}(\bar{\Omega}) \text { and }\left(t_{1}, t_{2}\right) \subseteq I^{ \pm}
\end{array}\right.
$$

In the following corollary, we give a particular case of Theorem 1 that we believe is of particular interest. More precisely we give a consequence of the theorem in the case where $u_{0} \geq 0, f \in L^{2}(\Omega)$ and $g \in L^{2}(\Gamma)$, which is interesting for the study of maximal solutions in case $\Omega=\mathbb{R}^{N}$ (cf. [12] and [16]).

Corollary 1. Assume that $u_{0} \in L^{1}(\Omega)^{+}, g \in L^{2}(\Gamma)$ and $f \in L^{2}(\Omega)$.

i) If $\int_{\Gamma} g+\int_{\Omega} f \geq 0$, then there exists a unique solution $u$ of $E_{1}\left(u_{0}, f, g\right)$ in the following sense:

$$
\left\{\begin{array}{l}
u \in \mathcal{C}\left([0, \infty) ; L^{1}(\Omega)^{+}\right), u(0)=u_{0} \text { a.e. in } \Omega, u>0 \text { a.e. in }(0, \infty) \times \Omega, \\
w:=\log (u) \in L_{l o c}^{2}\left(0, \infty ; H^{1}(\Omega)\right), \frac{d}{d t} \int_{\Omega} \xi u+\int_{\Omega} \nabla w \cdot \nabla \xi=\int_{\Gamma} g \xi+\int_{\Omega} f \xi \\
\text { in } \mathcal{D}^{\prime}(0,+\infty) \text { and } \xi \in \mathcal{C}^{1}(\bar{\Omega}) .
\end{array}\right.
$$

ii) If $\int_{\Gamma} g+\int_{\Omega} f<0$, then setting

$$
\tau=-\int_{\Omega} u_{0} /\left(\int_{\Gamma} g+\int_{\Omega} f\right),
$$

there exists a unique solution $u$ of $E_{1}\left(u_{0}, f, g\right)$ in the following sense:

$$
\left\{\begin{array}{l}
u \in \mathcal{C}\left([0, \infty) ; L^{1}(\Omega)^{ \pm}\right) \cap L^{2}(Q), u(0)=u_{0} \text { a.e. in } \Omega, u(\tau) \equiv 0 \text { in } \Omega, \\
u>0 \text { a.e. in }(0, \tau) \times \Omega, u<0 \text { a.e. in }(\tau, \infty) \times \Omega, \\
w:=\varphi_{1}(u) \in L_{l o c}^{2}\left((0, \tau) \cup(\tau, \infty) ; H^{1}(\Omega)\right), \\
\frac{d}{d t} \int_{\Omega} \xi u+\int_{\Omega} \nabla w \cdot \nabla \xi=\int_{\Gamma} g \xi+\int_{\Omega} f \xi \quad \text { in } \mathcal{D}^{\prime}\left(t_{1}, t_{2}\right) \\
\forall \xi \in \mathcal{C}^{1}(\bar{\Omega}) \text { and }\left(t_{1}, t_{2}\right) \subset(0, \tau) \cup(\tau, \infty) .
\end{array}\right.
$$

At last, let us give results that show how a sign-changing solution of $E_{\alpha}\left(u_{0}, f, g\right)$ for $\alpha<1$ converges, as $\alpha \uparrow 1$, to a solution of $E_{1}\left(u_{0}, f, g\right)$, which does not change the sign.

Theorem 2. Assume that $u_{0} \in L^{p}(\Omega)$, for $p>1, f \in L^{2}(Q), g \in L^{2}(\Sigma)$ and for $2-p<\alpha<1$, denote by $u_{\alpha}$ the solution of $E_{\alpha}\left(u_{0}, f, g\right)$. Then, there exists $\underline{u}_{0} \in L^{\infty}(\Omega)^{ \pm}$, such that, by taking a subsequence if necessary, we have

$$
u_{\alpha} \rightarrow \underline{u} \text { in } \mathcal{C}\left([\delta, T), L^{1}(\Omega)\right) \quad \text { for any } 0<\delta<T, \quad \text { as } \alpha \rightarrow 1,
$$


and $\underline{u}$ is the solution of $E_{1}\left(\underline{u}_{0}, f, g\right)$. Moreover, $\underline{u}_{0}$ satisfies

i) If $u_{0} \in L^{2}(\Omega)^{ \pm}$, then $\underline{u}_{0}=u_{0}$, and $\delta=0$ is admissible in (1.8).

ii) If $\int_{\Omega} u_{0}=0$, then $\underline{u}_{0} \equiv 0$.

iii) If $\int_{\Omega}^{\Omega} u_{0}>0$, then $0 \leq \underline{u}_{0} \leq u_{0}^{+}$a.e. in $\Omega$ and $\underline{u}_{0}=0$ a.e. in $\left[u_{0} \leq 0\right]$.

iv) If $\int_{\Omega} u_{0}<0$, then $-u_{0}^{-} \leq \underline{u}_{0} \leq 0$ a.e. in $\Omega$ and $\underline{u}_{0}=0$ a.e. in $\left[u_{0} \geq 0\right]$.

In particular we have

Corollary 2. Under the assumptions of Theorem 1, if $\int_{\Omega} u_{0}=\int_{\Omega} f(t)=\int_{\Gamma} g(t)=$ 0 a.e. $t \in[0, T)$, then

$$
u_{\alpha} \rightarrow 0 \quad \text { in } \mathcal{C}\left((0, T), L^{1}(\Omega)\right)
$$

as $\alpha \rightarrow 1$.

In the rest of this paper, we will prove the above results. In section 2, we point out the outline of the proof of Proposition 1, and we prove Theorem 1 and a part of Theorem 2 that correspond to the regular case, i.e. for nonnegative or nonpositive initial data. In section 3 we complete the proof of Theorem 2 in the singular case, i.e. for sign-changing initial data. At last, in the Appendix we prove two technical lemmas that we use in this paper.

\section{2.. The Limit of the FAST Diffusion AS $\alpha \uparrow 1$ AND THE LOGARITHMIC DIFFUSION}

In order to study the problem $E_{\alpha}\left(u_{0}, f, g\right)$ by using nonlinear semigroup theory (cf. [14] and 4]), we set $X=L^{1}(\Omega) \times L^{1}(\Gamma)$ endowed with the natural norm

$$
|(f, g)|_{X}=\|f\|_{L^{1}(\Omega)}+\|g\|_{L^{1}(\Gamma)} \quad \text { for }(f, g) \in X,
$$

and we consider, in $X$, the following Cauchy problem:

$$
\frac{d U}{d t}+\mathcal{A}_{\alpha} U=(f, g) \text { on }(0, \infty) \quad U(0)=U_{0},
$$

where $U_{0} \in \overline{\mathcal{D}\left(\mathcal{A}_{\alpha}\right)}$ and $\mathcal{A}_{\alpha}$ is the nonlinear operator defined in $X$ by

$$
\mathcal{A}_{\alpha}(v, 0)=(f, g) \Leftrightarrow\left\{\begin{array}{l}
v \in L^{1}(\Omega) ; f \in L^{1}(\Omega), g \in L^{1}(\Gamma) \\
w:=\varphi_{\alpha}(v) \in W^{1,1}(\Omega), \Delta w \in L^{1}(\Omega) \text { and } \\
\int_{\Omega}(\nabla w \cdot \nabla \xi+f \xi)=\int_{\Gamma} g \xi, \quad \forall \xi \in \mathcal{C}^{1}(\bar{\Omega})
\end{array}\right.
$$

Through the implicit discretization in time arising in nonlinear semigroup theory (cf. [14] and 44), the study of $E_{\alpha}\left(u_{0}, f, g\right)$ is closely related to the elliptic problem

$$
v=\nabla \cdot\left(v^{-\alpha} \nabla v\right)+f \text { on } \Omega, \quad v^{-\alpha} \nabla v \cdot \vec{n}=g \text { on } \partial \Omega
$$

with $f \in L^{1}(\Omega)$ and $g \in L^{1}(\Gamma)$. It is known (see for instance [20]) that for any $\alpha<1$, there exists a unique solution $v$ of (2.2) in the sense that

$$
\left\{\begin{array}{l}
v \in L^{1}(\Omega), \varphi_{\alpha}(v) \in W^{1,1}(\Omega), \\
\int_{\Omega} \nabla \varphi_{\alpha}(v) \cdot \nabla \xi=\int_{\Omega}(f-v) \xi+\int_{\Gamma} g \xi \\
\forall \xi \in \mathcal{C}^{1}(\bar{\Omega}) .
\end{array}\right.
$$


Moreover, we have

$$
\int_{\Omega} v=\int_{\Omega} f+\int_{\Gamma} g
$$

and if $v, \hat{v}$ are two solutions corresponding to $f, \hat{f} \in L^{1}(\Omega)$ and $g, \hat{g} \in L^{1}(\Gamma)$, then (cf. Proposition E in [5])

$$
\int_{\Omega}(v-\hat{v})^{+} \leq \int_{\Omega}(f-\hat{f})^{+}+\int_{\Gamma}(g-\hat{g})^{+}
$$

and

$$
\int_{\Omega}|v-\hat{v}| \leq \int_{\Omega}|f-\hat{f}|+\int_{\Gamma}|g-\hat{g}|
$$

so that the operator $\mathcal{A}_{\alpha}$ defined as above is m-T-accretive in $X$. Moreover, using the results of 5 . (cf. step 1 of the proof of Theorem I, page 220), we deduce that

$$
\overline{\mathcal{D}\left(\mathcal{A}_{\alpha}\right)}=L^{1}(\Omega) \times\{0\} \quad \text { for any } \alpha<1 .
$$

So, by using general results of nonlinear semigroup theory we have

Corollary 3. If $\alpha<1$, then for any $u_{0} \in L^{1}(\Omega)$, there exists a unique $u \in$ $\mathcal{C}\left([0, T) ; L^{1}(\Omega)\right)$ such that $U=(u, 0)$ is the mild solution of (2.1) with $U_{0}=\left(u_{0}, 0\right)$. Moreover,

$$
\int_{\Omega} u(t)=\int_{\Omega} u_{0}+\int_{0}^{t}\left(\int_{\Omega} f+\int_{\Gamma} g\right) \quad \text { for each } t \in[0, T)
$$

and we may define a mapping $S_{\alpha}\left(u_{0}, f, g\right) \in L^{1}(\Omega) \times X \rightarrow u \in \mathcal{C}\left([0, T) ; L^{1}(\Omega)\right)$ such that the $L^{1}$-comparison principle holds; i.e., for any $u_{01}, u_{02} \in L^{1}(\Omega), f_{1}, f_{2} \in$ $L^{1}(Q)$ and $g_{1}, g_{2} \in L^{1}(\Sigma)$, if $u_{i}=S_{\alpha}\left(u_{0 i}, f_{i}, g_{i}\right)$ for $i=1$, 2 , then

$$
\begin{aligned}
\frac{d}{d t} \int_{\Omega}\left(u_{1}(t)-u_{2}(t)\right)^{+} \leq & \int_{\Gamma}\left(g_{1}-g_{2}\right)^{+}+\int_{\Omega \cap\left[u_{1}(t)>u_{2}(t)\right]}\left(f_{1}(t)-f_{2}(t)\right) \\
& +\int_{\Omega \cap\left[u_{1}(t)=u_{2}(t)\right]}\left(f_{1}(t)-f_{2}(t)\right)^{+}
\end{aligned}
$$

in $\mathcal{D}^{\prime}(0, T)$

Proposition 2. If $\alpha<1, u_{0} \in L^{2-\alpha}(\Omega), f \in L^{2}(Q)$ and $g \in L^{2}(\Sigma)$, then $S_{\alpha}\left(u_{0}, f, g\right)$ is the unique solution of $E_{\alpha}\left(u_{0}, f, g\right)$ in the sense of Proposition 1 .

The proof of this Proposition follows in the same way as the proof of Proposition 3 of 19 . Recall that the main difficulty comes from the fact that we are dealing with nonhomogeneous Neumann boundary conditions, so that somewhere in the proof one can get only an a priori estimate on the gradient of $\varphi_{\alpha}(u)$ which is not sufficient when we regularize and go to the limit. So, in order to estimate $\varphi_{\alpha}(u)$ in $H^{1}(\Omega)$, we have used the structure of $\varphi_{\alpha}$ and the $L^{1}$-estimate of $u$ (cf. Lemma 4 of [19]). Note that, in [19], the boundary condition was considered to be independent of $t$ but this does not present many real difficulties. We omit the details of the proofs and leave them to the reader.

Now, we can focus on the existence and uniqueness of a solution of $E_{1}\left(u_{0}, f, g\right)$. We construct this solution as the limit of $u_{\alpha}$ as $\alpha \uparrow 1$. Since the solution of $E_{\alpha}\left(u_{0}, f, g\right)$ is the mild solution given by the nonlinear semigroup generated by $\mathcal{A}_{\alpha}$, it is natural to study the limit of this operator as $\alpha \rightarrow 1$. This is equivalent to 
studying the limit of a solution of (2.2), as $\alpha \rightarrow 1$, which is the aim of the following proposition:

Proposition 3. Let $f \in L^{1}(\Omega), g \in L^{1}(\Gamma)$ and for $0<\alpha<1$, let $v_{\alpha}$ be the solution of (2.2).

1) If $\int_{\Omega} f+\int_{\Gamma} g \neq 0$ and $s=\operatorname{Sign}\left(\int_{\Omega} f+\int_{\Gamma} g\right)$, then there exists a unique solution $v$ of

$$
\left\{\begin{array}{l}
v \in L^{1}(\Omega)^{ \pm}, \text {s } v>0 \text { a.e. on } \Omega, w:=\varphi_{1}(v) \in W^{1,1}(\Omega), \\
\int_{\Omega} \nabla w \cdot \nabla \xi=\int_{\Omega}(f-v) \xi+\int_{\Gamma} g \xi, \forall \xi \in \mathcal{C}^{1}(\bar{\Omega}) .
\end{array}\right.
$$

Moreover, as $\alpha \rightarrow 1$,

$$
v_{\alpha} \rightarrow v \quad \text { in } L^{1}(\Omega)
$$

and

$$
\tilde{\varphi}_{\alpha}^{s}\left(v_{\alpha}\right):=\varphi_{\alpha}\left(v_{\alpha}\right)-s /(1-\alpha) \rightarrow w \quad \text { in } W^{1,1}(\Omega) \text {-weak }
$$

2) If $\int_{\Omega} f+\int_{\Gamma} g=0$, then $v_{\alpha} \rightarrow 0$, in $L^{1}(\Omega)$, as $\alpha \rightarrow 1$.

In order to prove this proposition, we begin by proving the following result:

Lemma 1. If $f \in L^{\infty}(\Omega)$ and $g \in L^{\infty}(\Gamma)$, then $\left(v_{\alpha}\right)_{0<\alpha<1}$ is bounded in $L^{q}(\Omega)$, for any $q>2$.

Proof. We prove that

$$
\int_{\Omega}\left|v_{\alpha}\right|^{q} \leq C_{1}\left(\frac{2^{\frac{2-\alpha}{2}}}{|\Omega|^{(1-\alpha) 2}}\left(\left\|\left.f\right|_{L^{1}(\Omega)}+\right\| g \|_{L^{1}(\Gamma)}\right)^{\frac{2-\alpha}{2}}+C K_{\alpha}\right)^{\frac{2}{q-\alpha}}
$$

where

$$
\begin{aligned}
& C_{1}=C\left(\|f\|_{L^{\infty}(\Omega)}+\|g\|_{L^{\infty}(\Gamma)}\right) \max \left(|\Omega|^{\frac{1-\alpha}{q-\alpha}},|\Gamma|^{\frac{1-\alpha}{q-\alpha}}\right), \quad C_{2}=2^{\frac{q-\alpha}{2}} /|\Omega|^{\frac{q-1-\alpha}{2}}+C, \\
& k_{\alpha}=2\|v\|_{L^{1}(\Omega)}^{\frac{q-\alpha}{2}}+2\left(\frac{1}{2 C_{2}}\left(\frac{4}{C_{1}(q-\alpha)^{2}}\right)^{\frac{q-\alpha}{2}}\right)^{-\frac{1}{q-1-\alpha}}
\end{aligned}
$$

and $C$ denotes a constant depending only on $N$ and $\Omega$. Then, it is clear that $C_{1}, C_{2}$ and $K_{\alpha}$ are bounded in $m$ and the result of the lemma follows. Taking $\xi=\left|v_{\alpha}\right|^{q-2} v_{\alpha}$ as a test function in (2.3), we get

$$
\begin{aligned}
\int_{\Omega}|v|^{q}+\frac{4}{(q-\alpha)^{2}} \int_{\Omega}\left|\nabla v^{\frac{q-\alpha}{2}}\right|^{2} & =\int_{\Omega} f|v|^{q-2} v+\int_{\Gamma} g|v|^{q-2} v \\
& \leq C_{1}\left(\|v\|_{L^{q-\alpha}(\Omega)}+\|v\|_{L^{q-\alpha}(\Gamma)}\right) \\
& \leq C_{1}\left\|v^{\frac{q-\alpha}{2}}\right\|_{H^{1}(\Omega)}^{\frac{2}{q-\alpha}},
\end{aligned}
$$

where we drop the index $m$. Moreover, using Lemma 8 in the Appendix, we have

$$
\left\|v^{\frac{q-\alpha}{2}}\right\|_{L^{2}(\Omega)} \leq \frac{2^{\frac{q-\alpha}{2}}}{|\Omega|^{\frac{q-1-\alpha}{2}}}\|v\|_{L^{1}(\Omega)}^{\frac{q-\alpha}{2}}+C\left\|\nabla v^{\frac{q-\alpha}{2}}\right\|_{L^{2}(\Omega)}
$$


and

$$
\left\|v^{\frac{q-\alpha}{2}}\right\|_{H^{1}(\Omega)} \leq C_{2}\left(\|v\|_{L^{1}(\Omega)}^{\frac{q-\alpha}{2}}+\left\|\nabla v^{\frac{q-\alpha}{2}}\right\|_{L^{2}(\Omega)}\right) .
$$

So, (2.8) and (2.10) imply that

$$
\frac{4}{(q-\alpha)^{2}} \int_{\Omega}\left|\nabla v^{\frac{q-\alpha}{2}}\right|^{2} \leq C_{1}\left[C_{2}\left(\|v\|_{L^{1}(\Omega)}^{\frac{q-\alpha}{2}}+\left\|\nabla v^{\frac{q-\alpha}{2}}\right\|_{L^{2}(\Omega)}\right)\right]^{\frac{2}{q-\alpha}},
$$

which is equivalent to

$$
\left\|\nabla v^{\frac{q-\alpha}{2}}\right\|_{L^{2}(\Omega)}^{q-\alpha} \leq C_{2}\left(\frac{C_{1}(q-\alpha)^{2}}{4}\right)^{\frac{q-\alpha}{2}}\left(\|v\|_{L^{1}(\Omega)}^{\frac{q-\alpha}{2}}+\left\|\nabla v^{\frac{q-\alpha}{2}}\right\|_{L^{2}(\Omega)}\right) .
$$

Using Young, this implies that

$$
\left\|\nabla v^{\frac{q-\alpha}{2}}\right\|_{L^{2}(\Omega)} \leq \frac{\epsilon C_{2}\left(\frac{C_{1}(q-\alpha)^{2}}{4}\right)^{\frac{q-\alpha}{2}}\|v\|_{L^{1}(\Omega)}^{\frac{q-\alpha}{2}}+\epsilon^{-\frac{1}{q-1-\alpha}}}{1-\epsilon C_{2}\left(\frac{C_{1}(q-\alpha)^{2}}{2}\right)^{\frac{q-\alpha}{2}}}
$$

for any $\epsilon$ such that

$$
0<\epsilon<\frac{1}{C_{2}}\left(\frac{4}{C_{1}(q-\alpha)^{2}}\right)^{\frac{q-\alpha}{2}} .
$$

Taking $\epsilon=\frac{1}{2 C_{2}}\left(\frac{4}{C_{1}(q-\alpha)^{2}}\right)^{\frac{q-\alpha}{2}}$, we deduce that

$$
\left\|\nabla v^{\frac{q-\alpha}{2}}\right\|_{L^{2}(\Omega)} \leq K_{\alpha}
$$

so that (2.10) implies

$$
\left\|v^{\frac{q-\alpha}{2}}\right\|_{H^{1}(\Omega)} \leq \frac{2^{\frac{q-\alpha}{2}}}{|\Omega|^{\frac{q-1-\alpha}{2}}}\|v\|_{L^{1}(\Omega)}^{\frac{q-\alpha}{2}}+C K_{\alpha}
$$

and (2.7) follows by using (2.8).

Lemma 2. Under the assumptions of Proposition [3, $\left(v_{\alpha}\right)_{0<\alpha<1}$ is relatively compact in $L^{1}(\Omega)$.

Proof. First, we know (see for instance step 3 of the proof of Theorem B' [5]) that for all $\omega \Subset \Omega$, we have

$$
\lim _{|y| \rightarrow 0} \sup _{\alpha<1} \int_{\omega}\left|v_{\alpha}(x+y)-v_{\alpha}(x)\right| d x=0,
$$

so that $v_{\alpha}$ is relatively compact in $L^{1}(\omega)$, and since

$$
\left\|v_{\alpha}\right\|_{L^{1}(\Omega)} \leq\|f\|_{L^{1}(\Omega)}+\|g\|_{L^{1}(\Gamma)},
$$

then there exists $\alpha_{k} \rightarrow 1$ and $v \in L^{1}(\Omega)$ such that

$$
v_{\alpha_{k}} \rightarrow v \quad \text { a.e. } \Omega \text {. }
$$

Assume that $f \in L^{\infty}(\Omega)$ and $g \in L^{\infty}(\Gamma)$. Then using Lemma1, we deduce that $v_{\alpha}$ is bounded in $L^{p}(\Omega)$, with $p>2$. This implies that $v_{\alpha}$ is relatively compact in $L^{p}(\Omega)$ weak and then in $L^{1}(\Omega)$-weak. So, using (2.12) we deduce that $v_{\alpha}$ is relatively compact in $L^{1}(\Omega)$. If $f \in L^{1}(\Omega)$ and $g \in L^{1}(\Gamma)$, then we consider $f_{\varepsilon} \in L^{\infty}(\Omega)$, $g_{\varepsilon} \in L^{\infty}(\Gamma)$ such that $f_{\varepsilon} \rightarrow f$ in $L^{1}(\Omega)$ and $g_{\varepsilon} \rightarrow g$ in $L^{1}(\Gamma)$, as $\varepsilon \rightarrow 0$. Using 
the first step of the proof, we denote by $v_{\alpha \varepsilon}$ the corresponding solution which is convergent in $L^{1}(\Omega)$. Using (2.5) for $n \leq m \leq 1$, we have

$$
\begin{aligned}
\left\|v_{n}-v_{m}\right\|_{1} & \leq\left\|v_{n}-v_{n \varepsilon}\right\|_{1}+\left\|v_{m}-v_{m \varepsilon}\right\|_{1}+\left\|v_{n \varepsilon}-v_{m \varepsilon}\right\|_{1} \\
& \leq 2\left(\left\|f-f_{\varepsilon}\right\|_{1}+\left\|g-g_{\varepsilon}\right\|_{1}\right)+\left\|v_{n \varepsilon}-v_{m \varepsilon}\right\|_{1} .
\end{aligned}
$$

So,

$$
\limsup _{n \rightarrow 0}\left\|v_{n}-v_{m}\right\|_{1} \leq 2\left(\left\|f-f_{\varepsilon}\right\|_{1}+\left\|g-g_{\varepsilon}\right\|_{1}\right) \rightarrow 0, \text { as } \varepsilon \rightarrow 0
$$

and, we deduce that $v_{\alpha}$ is relatively compact in $L^{1}(\Omega)$.

Proof of Proposition 3. If $\int_{\Omega} f+\int_{\Gamma} g>0$, then $s=1$ and, as $\alpha \rightarrow 1, \tilde{\varphi}_{\alpha}^{1}(.) \rightarrow \log ($. in the graph sense. Since we can replace $\varphi_{\alpha}$ in (2.3) by $\tilde{\varphi}_{\alpha}^{s}$, then one can prove exactly in the same way as Theorem B of [5] that a limit $v$, through a subsequence of solution $v_{\alpha}$ (which exists by Lemma 22) is such that $v>0, \tilde{\varphi}_{\alpha}^{s}\left(v_{\alpha}\right) \rightarrow \log (v)=\varphi_{1}(v)$ in $W^{1,1}(\Omega)$-weak and $v$ satisfies

$$
\int_{\Omega} \nabla \varphi_{1}(v) \cdot \nabla \xi=\int_{\Omega}(f-v) \xi+\int_{\Gamma} g \xi, \forall \xi \in \mathcal{C}^{1}(\bar{\Omega})
$$

Using the symmetry $v_{\alpha} \rightarrow-v_{\alpha}$, the case $\int_{\Omega} f+\int_{\Gamma} g<0$ can be reduced to the previous case. The uniqueness follows by Proposition E of [5].

To prove the second part of the proposition, let us assume that $\int_{\Omega} f+\int_{\Gamma} g=0$. Again, according to [5], we have

$$
\left(\tilde{\varphi}_{\alpha}^{1}\left(v_{\alpha}\right)-C_{\alpha}\right)_{m \geq 1} \text { is bounded in } W^{1,1}(\Omega),
$$

where $C_{\alpha}=f \tilde{\varphi}_{\alpha}^{1}\left(v_{\alpha}\right)$. Using Lemma[2, there exists $\alpha_{k} \rightarrow 1$ such that $v_{k}:=v_{\alpha_{k}} \rightarrow v$ in $L^{1}(\Omega)$ and using (2.13) we have $\tilde{w}_{k}:=\tilde{\varphi}_{\alpha_{k}}^{1}\left(v_{k}\right)-C_{\alpha_{k}} \rightarrow \tilde{w}_{\infty}$ in $W^{1,1}(\Omega)$ and a.e. in $\Omega$. Since $v_{k}$ and $\tilde{w}_{k}$ converge in $L^{1}(\Omega)$, then $C_{\alpha_{k}}$ is not bounded. Indeed, if $C_{\alpha_{k}}$ is bounded, then $\tilde{\varphi}_{\alpha_{k}}^{1}\left(v_{k}\right)$ converges in $L^{1}(\Omega)$ and using that $\tilde{\varphi}_{\alpha}^{1}$ is monotonic and the fact that $\tilde{\varphi}_{\alpha}^{1} \rightarrow \log$ in the graph sense, as $\alpha \rightarrow 1$, we deduce that $v>0$, which contradicts the fact that $\int_{\Omega} v=\int_{\Omega} f+\int_{\Gamma} g=0$. So, using again (2.13), we deduce that $\tilde{\varphi}_{\alpha_{k}}^{1}\left(v_{k}\right) \rightarrow \pm \infty$ a.e. in $\Omega$. Now, since for $\alpha$ close enough to 1 , we have $\tilde{\varphi}_{\alpha}^{1}\left(v_{\alpha}\right) \leq v_{\alpha}^{+}$and $v_{\alpha}$ converges in $L^{1}(\Omega)$, then we deduce that $\tilde{\varphi}_{\alpha_{k}}^{1}\left(v_{k}\right) \rightarrow-\infty$ a.e. in $\Omega$, which implies that $v_{k} \rightarrow 0$.

Using Proposition 3, we have $\mathcal{A}_{\alpha} \rightarrow \mathcal{A}_{1}$ in the graph sense, where

$$
\mathcal{A}_{1}=B \cup\left\{((0,0),(f, g)) \in X \times X ; \int_{\Omega} f+\int_{\Gamma} g=0\right\}
$$


and $B$ is the T-accretive operator in $X$ defined by

$$
B(v, 0)=(f, g) \Leftrightarrow\left\{\begin{array}{l}
v \in L^{1}(\Omega)^{ \pm}, f \in L^{1}(\Omega), g \in L^{1}(\Gamma), \\
|v|>0 \text { a.e. in } \Omega, w:=\varphi_{1}(v) \in W^{1,1}(\Omega) \text { and } \\
\int_{\Omega} \nabla w \cdot \nabla \xi=\int_{\Omega} f \xi+\int_{\Gamma} g \xi \quad \forall \xi \in \mathcal{C}^{1}(\bar{\Omega}) .
\end{array}\right.
$$

Indeed, $\mathcal{A}_{1}$ being defined as above, for $(f, g) \in X$, we have

$$
(v, 0)+\mathcal{A}_{1}(v, 0) \ni(f, g) \Leftrightarrow\left\{\begin{array}{l}
v \in L^{1}(\Omega), \quad \int_{\Omega} v=\int_{\Omega} f+\int_{\Gamma} g \text { and } \\
\text { either } v \equiv 0 \text { or } v \text { is a solution of } S_{1}(f, g),
\end{array}\right.
$$

so that according to Proposition 3, there exists a unique solution $v$ of $(v, 0)+$ $\mathcal{A}_{1}(v, 0) \ni(f, g)$ and

$$
(v, 0)=X-\lim _{\alpha \rightarrow 1}\left(I+\mathcal{A}_{\alpha}\right)^{-1}(f, g)
$$

Moreover, we have

\section{Proposition 4.}

$$
\overline{\mathcal{D}\left(\mathcal{A}_{1}\right)}=L^{1}(\Omega)^{ \pm} \times\{0\},
$$

where $\overline{\mathcal{D}\left(\mathcal{A}_{1}\right)}$ denotes the closure in $L^{1}(\Omega)$ of the domain of $\mathcal{A}_{1}$.

Proof. From the definition of $\mathcal{A}_{1}$ it is clear that $\overline{\mathcal{D}\left(\mathcal{A}_{1}\right)} \subseteq L^{1}(\Omega)^{ \pm} \times\{0\}$. Let us prove that $L^{1}(\Omega)^{+} \times\{0\} \subseteq \overline{\mathcal{D}\left(\mathcal{A}_{1}\right)}$ and $L^{1}(\Omega)^{-} \times\{0\} \subseteq \overline{\mathcal{D}\left(\mathcal{A}_{1}\right)}$. It is clear that $(0,0) \in \overline{\mathcal{D}\left(\mathcal{A}_{1}\right)}$. Now, let $u \in L^{1}(\Omega)^{+} \cap L^{\infty}(\Omega)$ be such that $u \not \equiv 0$. We consider $u_{\varepsilon}$ to be the solution of

$$
u_{\varepsilon}-\varepsilon \Delta \log \left(u_{\varepsilon}\right)=u \text { in } \Omega, \quad u_{\varepsilon}^{-1} \nabla u_{\varepsilon} \cdot \vec{n}=0 \text { on } \partial \Omega .
$$

Since $\int_{\Omega} u_{\varepsilon}=\int_{\Omega} u>0$ and, as $\varepsilon \rightarrow 0$, the graph $\varepsilon \log ($.$) converges in the graph$ sense to the graph $\beta \equiv 0$, then by Theorem $\mathrm{B}$ of [5], we deduce that $u_{\varepsilon} \rightarrow u$ in $L^{1}(\Omega)$. So, since $\left(u_{\varepsilon}, 0\right) \in \overline{\mathcal{D}\left(\mathcal{A}_{1}\right)}$, for each $\varepsilon>0$, we deduce that $(u, 0) \in \overline{\mathcal{D}\left(\mathcal{A}_{1}\right)}$. At last, the proof of $L^{1}(\Omega)^{-} \times\{0\} \subseteq \overline{\mathcal{D}\left(\mathcal{A}_{1}\right)}$ follows by using the symmetry $u \rightarrow-u$ of the equation.

Corollary 4. For any $u_{0} \in L^{1}(\Omega)^{ \pm}$, there exists a unique $u \in \mathcal{C}\left([0, T) ; L^{1}(\Omega)\right)$, such that $U=(u, 0)$ is the mild solution of

$$
\frac{d U}{d t}+\mathcal{A}_{1} U=(f, g) \text { on }(0, \infty), \quad U(0)=\left(u_{0}, 0\right) .
$$

Moreover, $u$ satisfies (2.6) and we may define a mapping $S_{1}\left(u_{0}, f, g\right) \in L^{1}(\Omega)^{ \pm} \times$ $X \rightarrow u \in \mathcal{C}\left([0, T) ; L^{1}(\Omega)^{ \pm}\right)$such that the $L^{1}$-comparison principle holds. 
Using Proposition 1 with the Brézis-Pazy Theorem (cf. [9]), for the regular perturbations of nonlinear semigroups, we have

Corollary 5. Assume that $u_{0} \in L^{1}(\Omega)^{ \pm}$. If for $\alpha<1, f_{\alpha} \in L^{1}(Q), g_{\alpha} \in L^{1}(\Gamma)$ and $u_{0 \alpha} \in L^{1}(\Omega)$ are such that, as $\alpha \rightarrow 1, f_{\alpha} \rightarrow f$ in $L^{1}(Q), g_{\alpha} \rightarrow g$ in $L^{1}(\Sigma)$ and $u_{0 \alpha} \rightarrow u_{0}$ in $L^{1}(\Omega)$, then

$$
S_{\alpha}\left(u_{0 \alpha}, f_{\alpha}, g_{\alpha}\right) \rightarrow S_{1}\left(u_{0}, f, g\right) \quad \text { in } \mathcal{C}\left([0, T) ; L^{1}(\Omega)\right) .
$$

The next proposition leads to the existence and uniqueness of a weak solution of the diffusion equation with logarithmic nonlinearity and initial data $u_{0} \in L^{1}(\Omega)^{ \pm}$. To put to paper these results we need to introduce the following nonnegative function:

$$
j(r)= \begin{cases}r \log (r)-r+1 & \text { if } r>0, \\ 1 & \text { if } r=0 .\end{cases}
$$

Proposition 5. Let $u_{0} \in L^{1}(\Omega)^{+}$such that $j\left(u_{0}\right) \in L^{1}(\Omega), g \in L^{2}(\Sigma)$ and $f \in$ $L^{2}(Q)$. Assume that $\mu(t)>0$ for any $t \in[0, \tau]$. Then $u:=S_{1}\left(u_{0}, f, g\right)$ is the unique solution of $E_{1}\left(u_{0}, f, g\right)$ in $[0, \tau)$, in the following sense:

$$
\left\{\begin{array}{l}
u \in \mathcal{C}\left([0, \tau], L^{1}(\Omega)^{+}\right), u(0)=u_{0} \text { a.e. in } \Omega, \\
u>0 \text { a.e. in }(0, \tau) \times \Omega, \log (u) \in L^{2}\left(0, \tau ; H^{1}(\Omega)\right), \\
\frac{d}{d t} \int_{\Omega} \xi u+\int_{\Omega} \nabla \log (u) \cdot \nabla \xi=\int_{\Omega} f \xi+\int_{\Gamma} g \xi \\
\text { in } \mathcal{D}^{\prime}(0, \tau), \forall \xi \in \mathcal{C}^{1}(\bar{\Omega}) .
\end{array}\right.
$$

Using the symmetry $u \rightarrow-u$, we also have the following result:

Corollary 6. Let $u_{0} \in L^{1}(\Omega)^{-}$such that $j\left(-u_{0}\right) \in L^{1}(\Omega), g \in L^{2}(\Sigma)$ and $f \in$ $L^{2}(Q)$. Assume that $\mu(t)<0$ for any $t \in[0, \tau]$. Then $u:=S_{1}\left(u_{0}, f, g\right)$ is the unique solution of $E_{1}\left(u_{0}, f, g\right)$ in the following sense:

$$
\left\{\begin{array}{l}
u \in \mathcal{C}\left([0, \tau], L^{1}(\Omega)^{-}\right), u(0)=u_{0} \text { a.e. in } \Omega, \\
u<0 \text { a.e. in }(0, \tau) \times \Omega, \log (|u|) \in L^{2}\left(0, \tau ; H^{1}(\Omega)\right), \\
\frac{d}{d t} \int_{\Omega} \xi u-\int_{\Omega} \nabla \log (|u|) \cdot \nabla \xi=\int_{\Omega} f \xi+\int_{\Gamma} g \xi \\
\text { in } \mathcal{D}^{\prime}(0, \tau), \forall \xi \in \mathcal{C}^{1}(\bar{\Omega}) .
\end{array}\right.
$$

Remark 1. Note that in order for $u_{0}$ to satisfy the assumption of Proposition 5 or Corollary [6] it is enough that $u_{0} \in L^{p}(\Omega)^{ \pm}$for $p>1$.

Lemma 3. Let $u \in \mathcal{C}\left([0, T], L^{1}(\Omega)\right)$ such that $\inf _{t \in[0, T]} \int_{\Omega} u(t)>0$ and let $u_{\varepsilon} \in$ $\mathcal{C}\left([0, T], L^{1}(\Omega)\right)$ such that $u_{\varepsilon} \rightarrow u$ in $\mathcal{C}\left([0, T], L^{1}(\Omega)\right)$. If $\log \left(u_{\varepsilon}\right) \in L^{2}\left(0, T ; H^{1}(\Omega)\right.$, then there exists a constant $C$, such that

$$
\left\|\log \left(u_{\varepsilon}(t)\right)\right\|_{L^{2}(\Omega)} \leq C\left(\left\|\nabla \log \left(u_{\varepsilon}(t)\right)\right\|_{L^{2}(\Omega)}+\left\|u_{\varepsilon}(t)\right\|_{L^{2}(\Omega)}\right),
$$

for any $\varepsilon>0$ and $t \in[0, T]$.

Proof. First, one sees that using the Poincaré inequality, for any $K \subseteq \Omega$ and $w \in$ $H^{1}(\Omega)$, we have

$$
|K||f w| \leq C\left(\|\nabla w\|_{L^{2}(\Omega)}+\|w\|_{L^{2}(K)}\right),
$$


where $C$ depends only on $N$ and $\Omega$. Since there exists $\delta>0$ such that

$$
\inf _{t \in[0, T]} \int_{\Omega} u(t)>\delta>0,
$$

then $K(t)=[u(t)>\delta]$ satisfies

$$
|K(t)| \geq \inf _{t \in[0, T]}|K(t)|=: M>0 \quad \text { for any } t \in[0, T] .
$$

Now denote by $K_{\varepsilon}(t)=\left[u_{\varepsilon}(t)>\delta\right]$. Since $u_{\varepsilon}(t) \rightarrow u(t)$ in $L^{1}(\Omega)$, then from Fatou, we get

$$
|K(t)| \leq \liminf _{\varepsilon \rightarrow 0}\left|K_{\varepsilon}(t)\right| \quad \text { for any } t \in[0, T] .
$$

Applying (2.15) with $w=\log \left(u_{\varepsilon}(t)\right), K=K_{\varepsilon}(t)$ and using (2.16) and (2.17), we deduce that

$$
\left|f \log \left(u_{\varepsilon}(t)\right)\right| \leq \frac{C}{M}\left(\left\|\nabla \log \left(u_{\varepsilon}(t)\right)\right\|_{L^{2}(\Omega)}+\left\|\log \left(u_{\varepsilon}(t)\right)\right\|_{L^{2}\left(K_{\varepsilon}(t)\right)}\right)
$$

for any $\varepsilon>0$ and $t \in[0, T]$. On the other hand, since there exists $C^{\prime} \in \mathbb{R}$ independent of $\varepsilon$ and $t$, such that for $\varepsilon>0$ small enough and $t \in[0, T],\left|\log \left(u_{\varepsilon}(t)\right)\right| \leq$ $C^{\prime}\left|u_{\varepsilon}(t)\right|$ a.e. in $K_{\varepsilon}(t)$, then (2.18) implies that

$$
\left|f \log \left(u_{\varepsilon}(t)\right)\right| \leq C_{1}\left(\left\|\nabla \log \left(u_{\varepsilon}(t)\right)\right\|_{L^{2}(\Omega)}+\left\|u_{\varepsilon}(t)\right\|_{L^{2}(\Omega)}\right)
$$

and the result of the lemma follows by using the Poincaré inequality again.

Proof of Proposition 5. First, we show that $u$ satisfies (2.14). By the definition of $S_{1}\left(u_{0}, f, g\right), u(t)=L^{1}-\lim u_{\varepsilon}(t)$ uniformly for $t \in[0, T]$, where for $\varepsilon>0, u_{\varepsilon}$ is an $\varepsilon$-approximate solution corresponding to a subdivision $t_{0}=0<t_{1}<\ldots<t_{n-1}<$ $T=t_{n}$ with $t_{i}-t_{i-1}=\varepsilon, f_{1}, \ldots, f_{n} \in L^{2}(\Omega)$ and $g_{1}, \ldots, g_{n} \in L^{2}(\Gamma)$ with

$$
\sum_{i=1}^{n} \int_{t_{i-1}}^{t_{i}}\left(\left\|f(t)-f_{i}\right\|_{L^{1}(\Omega)}+\left\|g(t)-g_{i}\right\|_{L^{1}(\Gamma)}\right) d t \leq \varepsilon .
$$

That is, $u_{\varepsilon}$ is defined by $u_{\varepsilon}(0)=u_{0}, u_{\varepsilon}(t)=u_{i}$ for $\left.\left.t \in\right] t_{i-1}, t_{i}\right]$, where $u_{i} \in L^{1}(\Omega)$ satisfies

$$
\left(u_{i}, 0\right)+\varepsilon \mathcal{A}_{1}\left(u_{i}, 0\right)=\varepsilon\left(f_{i}, g_{i}\right)+\left(u_{i-1}, 0\right) .
$$

By definition of $\mathcal{A}_{1}$, we have

$$
\int_{\Omega} u_{i}=\int_{\Omega} u_{i-1}+i \varepsilon\left(\int_{\Omega} f_{i}+\int_{\Gamma} g_{i}\right)
$$

for any $i=0, \ldots, n$, so that

$$
\int_{\Omega} u_{i}=\int_{\Omega} u_{0}+\varepsilon \sum_{j=1}^{i}\left(\int_{\Omega} f_{j}+\int_{\Gamma} g_{j}\right)
$$

which implies that

$$
\begin{aligned}
\int_{\Omega} u_{\varepsilon}(t) & =\int_{\Omega} u_{0}+\int_{0}^{t}\left(\int_{\Omega} f_{\varepsilon}+\int_{\Gamma} g_{\varepsilon}\right) \\
& =: \quad \mu_{\varepsilon}(t) .
\end{aligned}
$$


FROM FAST TO VERY FAST DIFFUSION IN NONLINEAR HEAT EQUATION 5101

Since $u_{\varepsilon}$ converges to $u$ in $\mathcal{C}\left([0, \tau], L^{1}(\Omega)\right)$ and $\inf _{t \in[0, \tau]} \mu(t)>0$, then for each $\varepsilon$ small enough we have

$$
\inf _{t \in[0, \tau]} \mu_{\varepsilon}(t)>0
$$

and $\int_{\Omega} u_{i}=\mu_{\varepsilon}\left(t_{i}\right)>0$ for any $i=0,1, \ldots, n$. So, by definition of $\mathcal{A}_{1}$, this implies that $u_{i} \in L^{1}(\Omega), w_{i}:=\log \left(u_{i}\right) \in H^{1}(\Omega)$ and

$$
\int_{\Omega} \nabla w_{i} \cdot \nabla \xi=\int_{\Omega} f_{i} \xi+\int_{\Gamma} g_{i} \xi-\int \frac{u_{i}-u_{i-1}}{\varepsilon} \xi, \forall \xi \in \mathcal{C}^{1}(\bar{\Omega}) .
$$

Replacing $\xi$ by $w_{i}$ in (2.20), we get

$$
\begin{aligned}
\int_{\Omega} j\left(u_{i}\right)+\varepsilon \int_{\Omega}\left|\nabla w_{i}\right|^{2} \leq & \varepsilon\left(\int_{\Omega} f_{i} w_{i}+\int_{\Gamma} g_{i} w_{i}\right)+\int_{\Omega} j\left(u_{i-1}\right) \\
\leq & \varepsilon\left(\left\|f_{i}\right\|_{L^{2}(\Omega)}+\left\|g_{i}\right\|_{L^{2}(\Gamma)}\right)\left\|w_{i}\right\|_{H^{1}(\Omega)} \\
& +\int_{\Omega} j\left(u_{i-1}\right) .
\end{aligned}
$$

Adding (2.21) for $i=0, \ldots, n$, we deduce that $w_{\varepsilon}:=\log \left(u_{\varepsilon}\right)$ satisfies

$$
\begin{aligned}
\int_{\Omega} j\left(u_{\varepsilon}\right)+\int_{0}^{T} \int_{\Omega}\left|\nabla w_{\varepsilon}\right|^{2} \leq & \int_{0}^{\tau}\left(\left\|f_{\varepsilon}\right\|_{L^{2}(\Omega)}+\left\|g_{\varepsilon}\right\|_{L^{2}(\Gamma)}\right)\left\|w_{\varepsilon}\right\|_{H^{1}(\Omega)} \\
& +\int_{\Omega} j\left(u_{0}\right) .
\end{aligned}
$$

In addition, remember that

$$
\left\|u_{\varepsilon}(t)\right\|_{L^{1}(\Omega)} \leq\left\|u_{0}\right\|_{L^{1}(\Omega)}+\int_{0}^{T}\left(\left\|f_{\varepsilon}\right\|_{L^{1}(\Omega)}+\left\|g_{\varepsilon}\right\|_{L^{1}(\Gamma)}\right)
$$

for any $0 \leq t \leq \tau$, so that, using Lemma 3 and (2.19), we have

$$
\left\|w_{\varepsilon}(t)\right\|_{H^{1}(\Omega)} \leq C\left(1+\left\|\nabla w_{\varepsilon}(t)\right\|_{L^{2}(\Omega)}\right)
$$

with $C$ independent of $\varepsilon$ and $t$, and (2.22) implies

$$
\begin{aligned}
\int_{\Omega} j\left(u_{\varepsilon}\right)+\int_{0}^{T} \int_{\Omega}\left|\nabla w_{\varepsilon}\right|^{2} \leq & C \int_{0}^{\tau}\left(\left\|f_{\varepsilon}\right\|_{L^{2}(\Omega)}+\left\|g_{\varepsilon}\right\|_{L^{2}(\Gamma)}\right)\left(1+\left\|\nabla w_{\varepsilon}\right\|_{L^{2}(\Omega)}\right) \\
& +\int_{\Omega} j\left(u_{0}\right) \\
\leq & C\left(1+\left\|\nabla w_{\varepsilon}\right\|_{L^{2}(Q)}\right) \int_{0}^{\tau}\left(\left\|f_{\varepsilon}\right\|_{L^{2}(\Omega)}+\left\|g_{\varepsilon}\right\|_{L^{2}(\Gamma)}\right) \\
& +\int_{\Omega} j\left(u_{0}\right) .
\end{aligned}
$$

Using for instance Young, we deduce that $\nabla w_{\varepsilon}$ is bounded in $L^{2}(Q)$ and, by (2.23), $w_{\varepsilon}$ is bounded in $L^{2}\left(0, T ; H^{1}(\Omega)\right)$. So, there exists a subsequence that we denote 
again by $\varepsilon$ such that

$$
w_{\varepsilon} \rightarrow w \quad \text { weakly in } L^{2}\left(0, T ; H^{1}(\Omega)\right) \quad \text { as } \varepsilon \rightarrow 0 .
$$

Using a classical monotonic argument we deduce that $u>0$ and $w=\log (u)$ a.e. in $Q$. At last, let $\tilde{u}_{\varepsilon}$ be the function from $\left[0, t_{n}\right]$ into $L^{1}(\Omega)$ defined by $\tilde{u}_{\varepsilon}\left(t_{i}\right)=u_{i}$, where $\tilde{u}_{\varepsilon}$ is linear in $\left[t_{i-1}, t_{i}\right]$; for $\left.\xi \in \mathcal{C}^{1} \bar{Q}\right)$ with $\xi(T,.) \equiv 0$,

$$
\int_{0}^{T} \int_{\Omega} \tilde{u}_{\varepsilon} \xi_{t}+\int_{\Omega} u_{0} \xi(0, .)=\int_{0}^{T} \int_{\Omega} \nabla w_{\varepsilon} \cdot \nabla \xi+\int_{0}^{T} \int_{\Omega} f_{\varepsilon} \xi+\int_{0}^{T} \int_{\Gamma} g_{\varepsilon} \xi .
$$

Passing to the limit in (2.24) we get that $u$ is a solution of (2.14). To complete the proof, we have to show the uniqueness of the solution to (2.14). If $\left(u_{1}, w_{1}\right)$ and $\left(u_{2}, w_{2}\right)$ satisfy (2.14), then, by density, we have

$$
\int_{0}^{T} \int_{\Omega}\left(u_{1}-u_{2}\right) \xi_{t}+\nabla\left(w_{1}-w_{2}\right) \cdot \nabla \xi=0
$$

for all $\xi \in \mathcal{C}^{1}(\bar{Q})$ with $\xi(T,.) \equiv 0$ with $w_{1}=\varphi_{1}\left(u_{1}\right)$ and $w_{2}=\varphi_{1}\left(u_{2}\right)$ a.e. in $Q$. So, applying Lemma A in the Appendix of 7] with $H=L^{2}(\Omega), V=H^{1}(\Omega)$, $a(u, v)=\int \nabla u \cdot \nabla v, u=u_{1}-u_{2}$ and $v=w_{1}-w_{2}$, the uniqueness follows.

Proof of Theorem 1. First, we prove the uniqueness of a solution $u$ of (1.6). By definition, a solution $u(t)$ of (1.6) is perfectly defined in $I$, and by Proposition [5 and Corollary 6, $u$ is also perfectly defined in $[0, \inf I)$ if inf $I \neq 0$. Now, if $a<\tilde{a}<\tilde{b}<b$, with $a, b \in I$ and $(\tilde{a}, \tilde{b}) \subseteq I^{+}$(resp. $\left.(\tilde{a}, \tilde{b}) \subseteq I^{-}\right)$, then it is clear that $\mu(t)>0$ (resp. $\mu(t)<0$ ), for any $t \in[\tilde{a}, \tilde{b}]$, so that applying Proposition 5 (resp. Corollary [6), we find $u=S_{1}(u(\tilde{a}), f, g)$ on $(\tilde{a}, \tilde{b}) \times \Omega$. Then, using the contraction property of $S_{1}$, if $u_{1}, u_{2}$ are two solutions of (1.6), then

$$
\left\|u_{1}(t)-u_{2}(t)\right\|_{L^{1}} \leq\left\|u_{1}(\tilde{a})-u_{2}(\tilde{a})\right\|_{L^{1}}, \quad \forall a<\tilde{a} \leq t<b .
$$

Since $u_{i} \in \mathcal{C}\left([0, T) ; L^{1}(\Omega)\right)$ and $u_{i}(\tilde{a}) \rightarrow u_{i}(a)=0$ as $\tilde{a} \rightarrow a$, for $i=1,2$, then $u_{1}(\tilde{a})-u_{2}(\tilde{a}) \rightarrow 0$ in $L^{1}(\Omega)$ as $\tilde{a} \rightarrow a$ and we conclude that $u_{1}=u_{2}$ on $(a, b) \times \Omega$. This ends the proof of uniqueness. For the existence of a solution to (1.6), let $u=S_{1}\left(u_{0}, f, g\right)$. Being a mild solution and thanks to Corollary 4, we have $u \in$ $\mathcal{C}\left([0, T) ; L^{1}(\Omega)^{ \pm}\right), u(0)=u_{0}$ and $f u(t)=\mu(t)$. Then, it is clear that $u(t) \equiv 0$ for any $t \in I$. What's more, applying Proposition 5 and Corollary 6 in the interval $\left(t_{1}, t_{2}\right)$ such that $\left(t_{1}, t_{2}\right) \subset I^{ \pm}$, the proof of the theorem is complete.

\section{3.. The Singular Limit, AS $\alpha \uparrow 1$}

In section 2 , we proved that, as $\alpha \rightarrow 1$, the semigroup $S_{\alpha}$ is defined in $L^{1}(\Omega)$ and, as $\alpha \rightarrow 1, S_{\alpha}$ converges to the semigroup $S_{1}$, which is defined only on $L^{1}(\Omega)^{ \pm}$. So (cf. Corollary 5), if the initial data $u_{0}$ is in the domain of definition of $S_{1}$, i.e. $u_{0} \in L^{1}(\Omega)^{ \pm}$, then a solution of $E_{\alpha}\left(u_{0}, f, g\right)$ converges to a solution of $E_{1}\left(u_{0}, f, g\right)$. Now, since $E_{1}\left(u_{0}, f, g\right)$ has no solution if $u_{0}$ changes signs on $\Omega$, we wonder what is the limit of a solution of $E_{\alpha}\left(u_{0}, f, g\right)$, as $\alpha \rightarrow 1$ in this case. This is the aim of this section. Based on an idea of [18] (see also [6]), in order to study the limit of $E_{\alpha}\left(u_{0}, f, g\right)$ as $\alpha \rightarrow 1$, we first study the limit of the homogeneous evolution problem associated with $E_{\alpha}\left(u_{0}, f, g\right)$. So, we assume that $f \equiv 0, g \equiv 0$ and we 
study the limit of $S_{\alpha}\left(u_{0}, 0,0\right)$. In other words, we consider the homogeneous initialboundary-value problem

$$
\begin{cases}u_{t}=\nabla \cdot\left(u^{-\alpha} \nabla u\right) & \text { in } Q=(0, \infty) \times \Omega, \\ u^{-\alpha} \nabla u \cdot \vec{n}=0 & \text { on } \Sigma=(0, \infty) \times \Gamma, \\ u(0, .)=u_{0}(.) & \text { in } \Omega .\end{cases}
$$

Throughout this section, we denote by $u_{\alpha}$ the solution of (3.1) that is equal to $S_{\alpha}\left(u_{0}, 0,0\right)$, which we denote by $S_{\alpha}\left(u_{0}\right)$ to simplify the notation. We are interested in the limit of $u_{\alpha}$ as $\alpha \rightarrow 1$, without assuming any sign conditions on $u_{0}$.

Theorem 3. Assume that $u_{0} \in L^{p}(\Omega)$, for $p>1$.

(1) If $\int_{\Omega} u_{0}=0$, then $u_{\alpha} \rightarrow 0$ in $\mathcal{C}\left((0, \infty), L^{1}(\Omega)\right)$, as $\alpha \rightarrow 1$.

(2) If $\int_{\Omega} u_{0} \neq 0$ and $s=\operatorname{Sign}\left(\int_{\Omega} u_{0}\right)$, then there exists $\underline{u}_{0}$ such that $s \underline{u}_{0} \in$ $L^{2}(\Omega)^{+},-u_{0}^{-} \leq \underline{u}_{0} \leq u_{0}^{+}$a.e. in $\Omega, \underline{u}_{0}=0$ a.e. in $\left\{x \in \Omega ; s u_{0}(x) \geq 0\right\}$, and, by taking a subsequence if necessary, we have

$$
u_{\alpha} \rightarrow \underline{u} \text { in } \mathcal{C}\left((0, \infty) L^{1}(\Omega)\right), \quad \text { as } \alpha \rightarrow 1,
$$

where $\underline{u}$ is the solution of $E_{1}\left(\underline{u}_{0}, 0,0\right)$.

In order to prove Theorem 3 , we begin by the following results concerning the existence of a limit of a solution of $E_{\alpha}\left(u_{0}, 0,0\right)$.

Lemma 4. $\left(u_{\alpha}\right)_{0<\alpha<1}$ is relatively compact in $L^{1}(Q)$.

Proof. It is not difficult to see that $u_{\alpha}$ is bounded in $L^{\infty}(\Omega)$ and $\left(u_{\alpha}\right)^{1-\alpha} /(1-\alpha)$ is bounded in $L^{2}\left(0, T ; H^{1}(\Omega)\right)$. Then, $\left(u_{\alpha}\right)_{0<\alpha<1}$ is also bounded in $L^{2}\left(0, T ; H^{1}(\Omega)\right)$ and $\lim _{h \rightarrow 0} \int_{0}^{T} \int_{\omega}\left|u_{\alpha}(t, x+h)-u_{\alpha}(t, x)\right|=0$, for each $\omega \Subset \Omega$. So, thanks to Theorem 2 of [23], the result of the lemma follows.

Lemma 5. Assume that $u_{0} \in L^{p}(\Omega)$, for $p>1$, and $\int_{\Omega} u_{0} \geq 0$. Then, there exists a subsequence $\alpha_{k} \rightarrow 1$, such that

$$
u_{\alpha_{k}} \rightarrow \underline{u} \text { in } \mathcal{C}\left((0, \infty), L^{1}(\Omega)\right),
$$

$\underline{u} \in \mathcal{C}\left((0, \infty) ; L^{1}(\Omega)\right), \underline{u} \geq 0$ a.e. $(0, \infty) \times \Omega, \log (\underline{u}) \in L^{2}\left(0, \tau ; H^{1}(\Omega)\right)$ and

$$
\frac{d}{d t} \int_{\Omega} \xi \underline{u}+\int_{\Omega} \nabla \log (\underline{u}) \cdot \nabla \xi=0
$$

in $\mathcal{D}^{\prime}(0, \tau)$ and for any $\xi \in \mathcal{C}^{1}(\bar{\Omega})$.

Proof. Thanks to Lemma 4, let $\tau>0, \alpha_{k} \rightarrow 1$ and $\underline{u}_{\tau} \in L^{1}(\Omega)$, such that $u_{\alpha_{k}}(\tau) \rightarrow$ $\underline{u}_{\tau}$. Let us first prove that $\underline{u}_{\tau} \geq 0$ a.e. in $Q$. Thanks to [22], we know that $z_{\alpha}=$ $u_{\alpha}-f u_{0}$, is also a solution of

$$
\left\{\begin{array}{l}
z_{t}+\partial G_{\alpha}(z) \ni 0 \quad \text { in }(0, T) \\
z(0)=u_{0}-f u_{0}=: z_{0}
\end{array}\right.
$$


in $X^{*}$, the dual space of $X=\left\{z \in H^{1}(\Omega) ; \int_{\Omega} z=0\right\}$ and $G_{\alpha}$ is the functional defined on $X^{*}$ by

$$
G_{\alpha}(z)= \begin{cases}\int_{\Omega} j_{\alpha}\left(z+f u_{0}\right) & \text { if } j_{\alpha}\left(z+f u_{0}\right) \in L^{1}(\Omega) \\ +\infty & \text { otherwise }\end{cases}
$$

where

$$
j_{\alpha}(r)=\frac{|r|^{2-\alpha}}{(1-\alpha)(2-\alpha)}-\frac{r}{1-\alpha}+\frac{1}{2-\alpha},
$$

for any $r \in \mathbb{R}$. Thanks to [10] (Lemma 3.3, page 73), we have

$$
\begin{aligned}
\tau G_{\alpha}\left(z_{\alpha}(\tau)\right) & \leq \int_{0}^{\tau} G_{\alpha}\left(z_{\alpha}(s)\right) d s \\
& \leq \int_{0}^{\tau}\left(z_{\alpha}(s),-z_{\alpha}^{\prime}(s)\right)_{X^{*}} d s+\tau G_{\alpha}(0),
\end{aligned}
$$

which implies that

$$
\begin{aligned}
\tau \int_{\Omega} j_{\alpha}\left(u_{\alpha}((\tau))\right. & \leq \frac{1}{2}\left|u_{0}\right|_{X^{*}}+\tau \int_{\Omega} j_{\alpha}\left(f u_{0}\right) \\
& \leq \frac{1}{2}\left|u_{0}\right|_{X^{*}}+\tau|\Omega| j_{\alpha}\left(f u_{0}\right) .
\end{aligned}
$$

It is not difficult to see that, as $\alpha \rightarrow 1$, then $j_{\alpha}$ converges in the graph sense to $j_{1}$, given by

$$
j_{1}(r)= \begin{cases}+\infty & \text { if } r<0 \\ 0 & \text { if } r=0 \\ r \log r-r & \text { if } r>0\end{cases}
$$

Since $f u_{0} \geq 0$, then

$$
\liminf _{\alpha \rightarrow 1} \int_{\Omega} j_{\alpha}\left(u_{\alpha}(\tau)\right)<\infty,
$$

and since $u_{\alpha_{k}}(\tau) \rightarrow \underline{u}_{\tau}$, then using Fatou we deduce that $\underline{u}_{\tau} \geq 0$ a.e. in $\Omega$. Now, since $\underline{u}_{\tau} \geq 0$ and $u_{\alpha_{k}}(\tau) \rightarrow \underline{u}_{\tau}$, then applying Corollary $\underline{5}$ in $(\tau, \infty)$, we deduce that $u_{\alpha_{k}}(t) \rightarrow S_{1}\left(\underline{u}_{\tau}\right)(t-\tau)$ in $L^{1}(\Omega)$ uniformly for bounded $t \geq \tau$, and that $v():.=S_{1}\left(\underline{u}_{\tau}\right)(.-\tau)$ satisfies (3.3) in $\mathcal{D}^{\prime}(\tau, T)$ for any $\xi \in \mathcal{C}^{1}(\bar{\Omega})$. By a diagonal process we deduce that $u_{\alpha_{k}}$ is relatively compact in $\mathcal{C}\left((0, \infty), L^{1}(\Omega)\right)$ and there exists $\underline{u} \in \mathcal{C}\left((0, \infty), L^{1}(\Omega)^{+}\right)$such that $u_{\alpha_{k}}$ converges, through a subsequence, to $\underline{u}$ in $\mathcal{C}\left((0, \infty), L^{1}(\Omega)\right)$ and $\underline{u}$ satisfies $(\underline{3.3)})$ in $\mathcal{D}^{\prime}(\tau, T)$ for any $\xi \in \mathcal{C}^{1}(\bar{\Omega})$.

Lemma 6. Let $u_{0} \in L^{p}(\Omega)$, for $p>1, \int u_{0} \geq 0$ and $\underline{u}$ be given as in Lemma 5 . Then $\underline{u}_{0}:=L^{1}(\Omega)-\lim _{t \rightarrow 0} \underline{u}(t)$ is well defined and we have

(1) if $\int_{\Omega} u_{0}>0$, then $0 \leq \underline{u}_{0} \leq u_{0}^{+}$a.e. in $\Omega$ and $\underline{u}_{0}=0$ a.e. in $\left[u_{0} \leq 0\right]$,

(2) if $\int_{\Omega} u_{0}=0$, then $\underline{u} \equiv 0$ in $[0, \infty) \times \Omega$. 
Proof. First one sees that since $\underline{u} \in \mathcal{C}\left((0, \infty), L^{1}(\Omega)\right), \underline{u}>0$ a.e. in $(0, T) \times \Omega$ and satisfies (3.3) in $\mathcal{D}^{\prime}(\tau, T)$ for any $\xi \in \mathcal{C}^{1}(\bar{\Omega})$, then $\underline{u}$ is a classical solution and $\underline{u}_{0}:=L^{1}(\Omega)-\lim _{t \rightarrow 0} \underline{u}(t) \geq 0$ is well defined. In addition, since $u_{0} \leq u_{0}^{+}$, then $u_{\alpha}(t) \leq S_{\alpha}\left(u_{0}^{+}\right)(t)$, for each $t \geq 0$ and, letting $\alpha \rightarrow 1$, we deduce, by using Corollary 5. that $\underline{u}(t) \leq S_{1}\left(u_{0}^{+}\right)(t)$, for any $t \geq 0$. Letting $t \rightarrow 0$, we get $0 \leq \underline{u}(0) \leq u_{0}^{+}$, and the first part of the lemma follows. The second part of the lemma easily follows from the additional fact that $\int_{\Omega} \underline{u}=\int_{\Omega} u_{\alpha}(t)=\int_{\Omega} u_{0}$, for any $t \geq 0$.

Proof of Theorem 3. Now, it is clear that the first part of the proof follows by 2) of Lemma 6. As for the second part, this is a direct consequence of Lemma 5 and 1) of Lemma 6. Finally, one sees that the case $\int_{\Omega} u_{0}<0$ follows simply by using the symmetry $u \rightarrow-u$.

At last, since the solution of $E_{\alpha}\left(u_{0}, f, g\right)$ is given by $S_{\alpha}\left(u_{0}, f, g\right)$, then the proof of Theorem 2 is given by the following lemma.

Lemma 7. Let $u_{0} \in L^{\infty}(\Omega), u_{0} \geq 0, \underline{u}_{0}$ be given as in Lemma $\left[\right.$, $f \in L^{2}(Q)$ and $g \in L^{2}(\Gamma)$. Then, there exists a subsequence $\alpha_{k} \rightarrow 1$, such that we have

$$
S_{\alpha_{k}}\left(u_{0}, f, g\right) \rightarrow S_{1}\left(\underline{u}_{0}, f, g\right) \text { in } \mathcal{C}\left((0, T) ; L^{1}(\Omega)\right)
$$

as $\alpha_{k} \rightarrow 1$.

Proof. Let $0<\delta \leq t_{1}<t_{2}<\infty$ and denote by $f_{\delta}$ and $g_{\delta}$ the functions $f_{\delta}(s)=$ $f(s+\delta)$ and $g_{\delta}(s)=g(s+\delta)$, for a.e. $s \in[0, T-\delta)$. For all $t \in\left[t_{1}, t_{2}\right]$, we have

$$
\begin{aligned}
&\left\|S_{\alpha}\left(u_{0}, f, g\right)(t)-S_{1}\left(\underline{u}_{0}, f_{\delta}, g_{\delta}\right)(t)\right\|_{1} \\
& \leq\left\|S_{\alpha}\left(u_{0}, f, g\right)(t)-S_{\alpha}\left(S_{\alpha}\left(u_{0}\right)(\delta), f, g\right)(t-\delta)\right\|_{1} \\
&+\left\|S_{\alpha}\left(S_{\alpha}\left(u_{0}\right)(\delta), f_{\delta}, g_{\delta}\right)(t-\delta)-S_{1}\left(S_{1}(\underline{u})(\delta), f_{\delta}, g_{\delta}\right)(t-\delta)\right\|_{1} \\
&+\left\|S_{1}\left(S_{1}\left(\underline{u}_{0}\right)(\delta), f_{\delta}, g_{\delta}\right)(t-\delta)-S_{1}\left(\underline{u}_{0}, f, g\right)(t)\right\|_{1} .
\end{aligned}
$$

Keeping in mind the semigroup property of $S_{\alpha}$, we get

$$
S_{\alpha}\left(u_{0}, f, g\right)(t)=S_{\alpha}\left(S_{\alpha}\left(\left(u_{0}, f, g\right)(\delta), f, g\right), f_{\delta}, g_{\delta}\right)(t-\delta)
$$

for any $t \geq \delta$, so that using the $L^{1}$ contraction property of $S_{\alpha}$, for $0 \leq m \leq 1$, we have

$$
\begin{array}{r}
\left\|S_{\alpha}\left(u_{0}, f, g\right)(t)-S_{1}\left(\underline{u}_{0}, f, g\right)(t)\right\|_{1} \leq\left\|S_{\alpha}\left(u_{0}, f, g\right)(\delta)-S_{\alpha}\left(u_{0}\right)(\delta)\right\|_{1} \\
+\left\|S_{\alpha}\left(S_{\alpha}\left(u_{0}\right)(\delta), f_{\delta}, g_{\delta}\right)(t-\delta)-S_{1}\left(S_{1}\left(\underline{u}_{0}\right)(\delta), f_{\delta}, g_{\delta}\right)(t-\delta)\right\|_{1} \\
+\left\|S_{1}\left(\underline{u}_{0}\right)(\delta)-S_{1}\left(\underline{u}_{0}, f, g\right)(\delta)\right\|_{1} .
\end{array}
$$

Thanks to Corollary 3 and Corollary 1, we have

$$
\left\|S_{\alpha}\left(u_{0}, f, g\right)(\delta)-S_{\alpha}\left(u_{0}\right)(\delta)\right\|_{1} \leq \int_{0}^{\delta}\left(\int_{\Omega}|f|+\int_{\Gamma}|g|\right)
$$


and

so that

$$
\left\|S_{1}\left(\underline{u}_{0}, f, g\right)(\delta)-S_{1}\left(u_{0}\right)(\delta)\right\|_{1} \leq \int_{0}^{\delta}\left(\int_{\Omega}|f|+\int_{\Gamma}|g|\right),
$$

$$
\begin{aligned}
& \left\|S_{\alpha}\left(u_{0}, f, g\right)(t)-S_{1}\left(\underline{u}_{0}, f, g\right)(t)\right\|_{1} \leq 2 \int_{0}^{\delta}\left(\int_{\Omega}|f|+\int_{\Gamma}|g|\right) \\
& \quad+\left\|S_{\alpha}\left(S_{\alpha}\left(u_{0}\right)(\delta), f_{\delta}, g_{\delta}\right)(t-\delta)-S_{1}\left(S_{1}\left(\underline{u}_{0}\right)(\delta), f_{\delta}, g_{\delta}\right)(t-\delta)\right\|_{1} .
\end{aligned}
$$

From Lemma 5 there exists $\alpha_{k} \rightarrow 1$, such that

$$
S_{\alpha_{k}}\left(u_{0}\right)(\delta) \rightarrow S_{1}\left(\underline{u}_{0}\right)(\delta) \quad \text { in } L^{1}(\Omega)
$$

and since $S_{1}\left(\underline{u}_{0}\right)(\delta) \geq 0$, then by Corollary 5 .

$$
S_{\alpha_{k}}\left(S_{\alpha_{k}}\left(u_{0}\right)(\delta), f, g\right)(t-\delta) \rightarrow S_{1}\left(S_{1}\left(\underline{u}_{0}\right)(\delta), f, g\right)(t-\delta) \quad \text { in } L^{1}(\Omega) .
$$

So, (3.5) implies that

$$
\lim _{\alpha_{k} \rightarrow \infty} \sup _{t \in\left[t_{1}, t_{2}\right]}\left\|S_{\alpha_{k}}\left(u_{0}, f, g\right)(t)-S_{1}\left(\underline{u}_{0}, f, g\right)(t)\right\|_{1} \leq 2 \int_{0}^{\delta}\left(\int_{\Omega}|f|+\int_{\Gamma}|g|\right)
$$

and, since $\delta$ is arbitrary in (3.7), then the result of the lemma follows, by letting $\delta$ go to 0 .

Proof of Theorem 2, Now, the theorem is a simple consequence of Lemma 7 and Theorem 3

\section{4.. Appendix}

Lemma 8. There exists $C=C(N, \Omega)$ such that for any $1 \leq p<\infty$ and $z \in L^{1}(\Omega)$ such that $|z|^{q-2} z=: z^{p} \in H^{1}(\Omega)$, we have

$$
\left\|z^{p}\right\|_{L^{2}(\Omega)} \leq \frac{2^{p}}{|\Omega|^{q-2 / 2}}\|z\|_{L^{1}(\Omega)}^{p}+C\left\|\nabla z^{p}\right\|_{L^{2}(\Omega)}
$$

Proof. Set $w=z^{p}$. Then using the Poincaré inequality, we have

$$
|f w| \leq \frac{1}{|K|^{1 / 2}}\left(C\|\nabla w\|_{L^{2}(\Omega)}+\|w\|_{L^{2}(K)}\right)
$$

for any $k \subseteq \Omega$ with $|K| \neq 0$, where $f w=\frac{1}{|\Omega|} \int_{\Omega} w$, and we have

$$
\begin{aligned}
\|w\|_{L^{2}(\Omega)} & \leq C\|\nabla w\|_{L^{2}(\Omega)}+|\Omega|^{1 / 2}|f w| \\
& \leq C\left(1+\left(\frac{|\Omega|}{|K|}\right)^{1 / 2}\|\nabla w\|_{L^{2}(\Omega)}+\left(\frac{|\Omega|}{|K|}\right)^{1 / 2}\|w\|_{L^{2}(K)} .\right.
\end{aligned}
$$

Taking $K=[|z|<\lambda]$, and using the fact that

$$
\begin{aligned}
|K| & =|\Omega|-|[|z| \geq \lambda]| \\
& \geq|\Omega|-\frac{1}{\lambda}\|z\|_{L^{1}(\Omega)},
\end{aligned}
$$


we get

$$
\left\|z^{p}\right\|_{L^{2}(\Omega)} \leq \lambda^{p}|\Omega|^{\frac{1}{2}}+C\left\{\left(\frac{\lambda|\Omega|}{\lambda|\Omega|-\|z\|_{L^{1}(\Omega)}}\right)^{\frac{1}{2}}+1\right\}\left\|\nabla z^{p}\right\|_{L^{2}(\Omega)}
$$

for all $\lambda>\frac{1}{|\Omega|}\|z\|_{L^{1}(\Omega)}$. Then, taking for instance $\lambda=\frac{2}{|\Omega|}\|z\|_{L^{1}(\Omega)}$, the result follows.

\section{Some comments:}

- Recall that in $\mathbb{R}^{N}$, nonnegative maximal solutions of (1.1) with $f \equiv 0$ and $\alpha=1$ were constructed in [16] as limits, as $R \rightarrow \infty$, of solutions in a ball $B(R)$, of radius $R>0$, with nonhomogeneous Neumann boundary condition $g=4 \pi$. These solutions exist up to the maximal time $\frac{1}{4 \pi} \int_{\Omega} u_{0}$, given by (1.7). So, in the case where $f \not \equiv 0$, one can construct by using Corollary 1 nonnegative maximal solutions up to $\tau=\int_{\Omega} u_{0} /\left(4 \pi+\int_{\Omega} f\right)$.

- There is a wide literature concerning the nonlinear evolution problem of type (1.1), i.e. $u_{t}-\Delta \varphi(u)=f$, with maximal monotone graph $\varphi$. But there are only a few results combining the range of $\varphi$ not equal to $\mathbb{R}$ and nonhomogeneous Neumann boundary conditions. The case $\varphi(r)=\log (r)$ is one typical example that we have studied in this paper. In a forthcoming paper [1, we will study this kind of question for a large class of maximal monotone graphs $\varphi$ and general operators of the Leray-Lions type instead of the Laplacian.

- Assume that $f \equiv 0$ and $g \equiv 0$. If $\int_{\Omega} u_{0}>0$ (resp. $\left.\int_{\Omega} u_{0}<0\right)$, it is known that a solution of $E_{\alpha}\left(u_{0}, f, g\right)$ may be a sign-changing function, at least for small $t$. In the present paper, Theorem 2 implies that when $\alpha$ reaches the limit 1 , the negative (resp. positive) part of $u_{\alpha}$ should disappear and a boundary layer appears at time $t=0$. Note also that in Theorem 2 , the initial boundary layer is not characterized in the case where $\int_{\Omega} u_{0} \neq 0$. However, the properties given for it enable one to characterize it in some particular cases, for instance if $u_{0}$ is radial and symmetric (one can see for instance 28] for $N=1$ ). We did not get into this question in this paper. It may be treated in forthcoming papers.

\section{ACKNOWLEDGEMENTS}

The author thanks L. Guyot for reviewing the English for the paper.

\section{REFERENCES}

[1] F. Andreu, N. Igbida, J. Mazon and J. Toledo. $L^{1}$ Existence and Uniqueness Results for Quasi-linear Elliptic Equations with Nonlinear Boundary Conditions. Ann. Inst. Henri Poincaré Anal. Non Linéaire 24: 61-89, 2007. MR2286559 (2007j:35056)

[2] D.G. Aronson. The porous medium equation, CIME Lectures, In some problems in nonlinear diffusion. In K. Kirchgassner H. Amann, N. Bazely, editors, Lecture Notes in Mathematics 1224, Springer-Verlag, New York, 1986. MR877986 (88a:35130) 
[3] Ph. Bénilan, L. Boccardo, and M. Herrero. On the limit of solution of $u_{t}=\Delta u^{m}$ as $m \rightarrow \infty$. In M. Bertch et al., editor, Proceedings Int. Conf., Torino, 1989. Some Topics in Nonlinaer PDE's.

[4] Ph. Bénilan, M.G. Crandall, and A. Pazy. Evolution Equation Governed by Accretive Operators (book to appear).

[5] Ph. Bénilan, M.G. Crandall, and P. Sacks. Some $L^{1}$ existence and dependence results for semilinear elliptic equations under nonlinear boundary conditions. Appl. Math. Optim., 17:203-224, 1988. MR922980 (89d:35055)

[6] Ph. BÉnilan and N. IgBida. Singular limit for perturbed nonlinear semigroup. Comm. Applied Nonlinear Anal., 3(4):23-42, 1996. MR.1420283 (97k:34088)

[7] Ph. BÉnilan and N. IgBida. The Mesa problem for Neumann boundary value problem. accepted in Jour. Func. Anal.

[8] J. G. Berryman and C. J. Holland. Asymptotic behavior of the nonlinear diffusion equation $n_{t}=\left(n^{-1} n_{x}\right)_{x}$. J. Math. Phys., 23, 983-987, 1982. MR659997 (83m:35070)

[9] H. BrÉzis and A. PAZY. Convergence and Approximation of Semigroups of Nonlinear Operators in Banach Spaces. J. Funct. Anal., 9:63-74, 1972. MR0293452 (45:2529)

[10] H. BRÉZIS. Monotonicity methods in Hilbert spaces and some applications to nonlinear partial differential equations. In E. Zarantonello, editor, Contribution to Nonlinear Functional Analysis. Academic Press, 1971, pp. 101-156. MR0394323 (52:15126)

[11] L. A. Caffarelli and A. Friedman. Continuity of the density of a gas flow in a porous medium. Trans. Amer. Math. Soc., 252:99-113, 1979. MR0534112 (80i:35090)

[12] J.R. Esteban, A. Rodriguez, and J. L. VÁzquez. A nonlinear heat equation with singular diffusivity. Comm. Partial Diff. Eqs., 13:985-1039, 1988. MR944437 (89h:35167)

[13] J.R. Esteban, A. Rodriguez, and J. L. VÁzquez. The maximal solution of the logarithmic fast diffusion equation in two space dimensions. Adv. Differential Equations, 2(6):867-894, 1997. MR1606339 (98k:35094)

[14] L. C. Evans. Appplication of nonlinear semigroup theory to certain partial differential equations. In M.G. CRANDALL, editor, Nonlinear Evolution Equations, Academic Press, New York, 1978. MR513818 (81b:47078)

[15] R.S. Hamilton. The Ricci flow on surfaces. Contemporary Math., 71:237-262, Amer. Math. Soc., Providence, RI, 1988. MR954419 (89i:53029)

[16] K.M. HuI. Existence of solutions of the equation $u_{t}=\Delta \log u$. Nonlinear Anal. TMA, 37:875-914, 1999. MR1695083 (2000c:35131)

[17] K.M. Hui. Singular limit of solutions of the equation $u_{t}=\Delta\left(\frac{u^{m}}{m}\right)$ as $m \rightarrow \infty$. Pacific Jour. Math., 187:297-316, 1999. MR1675029(99j:35096)

[18] N. IgBida. Limite singulière de problèmes d'évolution non linéaires. Thèse de doctorat, Université de Franche-Comté, 1997.

[19] N. IGBida. The mesa-limit of the porous medium equation and the Hele-Shaw problem. Diff. Integral Equations, 15(2):129-146, 2002. MR1870466 (2002m:35119)

[20] N. IGBida. The Hele-Shaw problem with dynamical boundary conditions. preprint.

[21] H. P. McKean. The central limit theorem for Carleman's equation. Israel J. Math, 21:54-92, 1975. MR0423553(54:11529)

[22] No. KenMOCHI. Neumann problems for a class of nonlinear degenerate parabolic equations. Diff. Integral Equations, 3(2):253-273, 1990. MR1025177 (91d:35120)

[23] S. N. Kruzhkov. Result Concerning the Nature of the Continuity of Solutions of Parabolic Equations and Some of Their Applications. Mat. Zametki, 6(1):97-108, 1969.

[24] T.G. KuRTz. Convergence of sequences of semigroups of nonlinear operators with an application to gas kinetics. Trans. A.M.S., 186:259-272, 1974. MR0336482 (49:1256)

[25] P.L. Lions, P.E. Souganidis, and J.L. VÁzquez. The relation between the porous medium and the eikonal equations in several space dimensions. Revista Mat. Iberoamericana, 3(3):275310, 1987. MR996819 (90g:35075)

[26] L.A. Peletier. The porous medium equation. In K. Kirchgassner H. Amann, N. Bazely, editors, In application of nonlinear analysis in the physical sciences, Boston, 1981. Pitnam.

[27] Ph. Rosenau. Fast and superfast diffusion processes. Physical Rev. Let, 74(7), 1056-1059, 1995.

[28] A. Rodriguez and J.L. VÁzquez. Obstructions to existence in fast-diffusion equations. preprint. 
FROM FAST TO VERY FAST DIFFUSION IN NONLINEAR HEAT EQUATION

[29] L.F. Wu. A new result for the porous medium equation derived from the Ricci flow. Bull. Amer. Math. Soc., 28:90-94, 1993. MR1164949 (93f:58245)

[30] L.F. WU. The Ricci flow on complete $\mathbb{R}^{2}$. Comm. in Analysis and Geometry, 1:439-472, 1993. MR:1266475 (95d:53043)

LAmfa, CNRS-UmR 6140, Université de Picardie Jules Verne, 33 rue Saint Leu, 80038 Amiens, France

E-mail address: noureddine.igbida@u-picardie.fr 